\title{
Physics Results From Polarized DIS
}

\author{
Gordon P. Ramsey \\ Loyola University of Chicago, Chicago, IL 60626, USA \\ Argonne National Laboratory, IL 60439, USA \\ e-mail: gpr@hep.anl.gov
}

\begin{abstract}
We have extracted polarized nucleon distributions from recent data at CERN, SLAC and DESY. The flavor-dependent valence and sea quark spin distributions are determined for each experiment. We take into account possible differences in the up and down sea distributions, and assume that the strange sea contribution is suppressed by mass effects. Physics results determined from different experiments are compared, including higher order corrections.
\end{abstract}

\section{Polarized Quark Models}

Recently, the Spin Muon Collaboration (SMC) group from CERN [1] and the experimental groups from SLAC [2] and DESY [3] measured $A_{1}$ and $g_{1}$ to low $x$ for the proton, neutron and deuteron in deep-inelastic scattering (DIS). In these experiments, they have improved statistics and minimized the systematic errors. The measurement of $g_{1}^{p, n, d}$ provides a means to extract the polarized quark contributions to proton spin, using the DIS data and sum rules.

This summary is a similar presentation as that of reference [4], but modified to correctly include the recent calculation of the singlet QCD corrections and to introduce a further breaking of the symmetry of the polarized sea.

The polarized valence quark distributions are obtained from the unpolarized ones by starting with a modified 3-quark model. [4,5] Here, the polarized valence distributions are a linear combination of the unpolarized distributions with a free parameter to be determined by the sum rules. The flavor symmetry of the sea is broken by assuming that the polarization of the heavier strange quarks is suppressed and that there is a possibility that the polarized down quark distribution is different from the polarized up quark distribution, analogous to the unpolarized case. The sea distributions are then related by:

$$
c \Delta \bar{u}(x)=c \Delta u(x)=\Delta \bar{d}(x)=\Delta d(x)=[1+\epsilon] \Delta \bar{s}(x)=[1+\epsilon] \Delta s(x),
$$

where $c$ is the enhancement of $\Delta \bar{d}$ over $\Delta \bar{u}$ and $\epsilon$ is a measure of the increased difficulty in polarizing the strange quarks. For the symmetric sea, $c=1$ and

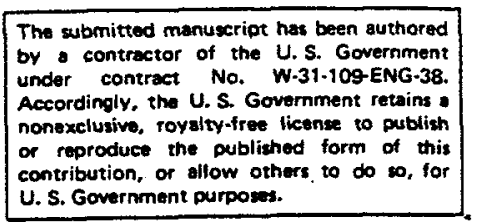

This work supported in part by the U.S. Department of Energy, Division of High Energy Physics, Contract W-31-109-ENG-38. 


\section{DISCLAIMER}

Portions of this document may be illegible in electronic image products. Images are produced from the best available original document. 


\section{DISCLAIMER}

This report was prepared as an account of work sponsored by an agency of the United States Government. Neither the United States Government nor any agency thereof, nor any of their employees, make any warranty, express or implied, or assumes any legal liability or responsibility for the accuracy, completeness, or usefulness of any information, apparatus, product, or process disclosed, or represents that its use would not infringe privately owned rights. Reference herein to any specific commercial product, process, or service by trade name, trademark, manufacturer, or otherwise does not necessarily constitute or imply its endorsement, recommendation, or favoring by the United States Government or any agency thereof. The views and opinions of authors expressed herein do not necessarily state or reflect those of the United States Government or any agency thereof. 
$\epsilon=0$, which is allowed as a possibility. In this model of the polarized quark distributions, the unknown factors reduce to the possible set: $\Delta q_{v}, \Delta \bar{u}, \epsilon$ and c. Knowing these will determine the full flavor dependence of nucleon spin.

\section{Constraints}

In order to compare the constraints in determining these factors more explicitly to the evolution equations, I will write them in terms of the their non-singlet and singlet combinations. These constraints are given by linear combinations of the non-vanishing axial-vector matrix elements $a_{3}, a_{8}$ and $a_{0}$. The first is related to the Bjorken sum rule (BSR), which is a fundamental test of QCD. In terms of the model described above, the BSR can be reduced to:

$$
\int_{0}^{1}\left(g_{1}^{p}-g_{1}^{n}\right) d x=\frac{1}{6} \int_{0}^{1}\left[\Delta u_{v}\left(x, Q^{2}\right)-\Delta d_{v}\left(x, Q^{2}\right)+2(1-c) \Delta \bar{u}\right] d x=\frac{a_{3}}{6}\left(1-\alpha_{1}^{N S}\right),
$$

where the non-singlet QCD corrections have been calculated to $O\left(\alpha_{s}^{3}\right)$. [6]

The non-singlet quark combination is a linear combination of the BSR and the parton interpretation of $a_{8}$, whose value is determined by hyperon decay. This combination is defined as

$$
\Delta q^{N S} \equiv \sum_{i=1}^{n}\left[\frac{e_{i}^{2}}{\left\langle e_{i}^{2}\right\rangle}-1\right] \Delta q_{i}=\Delta u_{t o t}-\frac{1}{2}\left[\Delta d_{t o t}+\Delta s_{t o t}\right] .
$$

In our model, this can be represented as:

$$
\Delta q^{N S}=1.5\left\langle\Delta q_{v}\right\rangle+\left[2-\left(\frac{2+\epsilon}{1+\epsilon}\right) c\right]\langle\Delta \bar{u}\rangle=\frac{1}{4}\left[3 a_{3}+a_{8}\right]\left(1-\alpha_{s}^{N S}\right) .
$$

The model of $\Delta G$ that is used has an effect on the quark distributions through the gluon axial anomaly, [7] which has the general form: $\Gamma\left(Q^{2}\right)=$ $\frac{\alpha_{\alpha}\left(Q^{2}\right)}{2 \pi} \int_{0}^{1} \Delta G\left(x, Q^{2}\right) d x$. We have used two models for $\Delta G$ : (1) $\Delta G=x G$ and (2) $\Delta G=0$. The second model sets the anomaly term equal to zero, which represents the gauge independent factorization, and is the model to be compared to the lattice results of the polarized distributions. We believe that present data imply that $\Delta G$ is limited at low $Q^{2}$. The matrix element $a_{0}$ is related to the total spin carried by the quarks in the proton, modified by the axial gluon anomaly: $\Delta q_{\text {tot }}=a_{0}+\Gamma$.

The integrated polarized structure function, $I^{p(n)} \equiv \int_{0}^{1} g_{1}^{p(n)}(x) d x$, is related to the polarized quark distributions by

$$
I^{p(n)}=\frac{1}{18}\left(1-\alpha_{s}^{\text {corr }}\right)\left\langle\left[4(1) \Delta u_{t o t}+1(4) \Delta d_{t o t}+\left(\Delta s_{t o t}\right)\right]\right\rangle .
$$




$$
=\left[+(-) \frac{a_{3}}{12}+\frac{a_{8}}{36}\right]\left(1-\alpha^{N S}\right)+\frac{a_{0}}{9}\left(1-\alpha_{8}^{s}\right),
$$

where the singlet QCD corrections, $\alpha_{a}^{s}$, have been caluclated to $O\left(\alpha_{s}^{3}\right)$. [8] The higher twist corrections [9] are negligible at the $Q^{2}$ values of the data.

\section{Results}

In order to check the validity of the BSR, we have derived an effective $I^{p}$ from either $I^{\mathrm{n}}$ or $I^{d}$ obtained from neutron or deuteron DIS data by using the BSR form in equation (2) and the relation: $I^{d}=\int_{0}^{1} g_{1}^{d}(x) d x=\frac{1}{2}\left[I^{p}+I^{n}\right](1-$ $\left.\frac{3}{2} \omega_{D}\right)$, where $\omega_{D}$ is the probability that the deuteron will be in a $D$-state. The BSR is tested by comparing this effective $I^{p}$ for all data. Equations (2) through (5) are used to extract the flavor dependent information on the total flavor dependent contributions to proton spin. Results are given in Table I. These particular contributions are independent of the parameter $c$, which affects the sea distributions and valence distributions separately. Each of the extracted distributions below are valid to within $\pm 10 \%$, including both theoretical and experimental uncertainties.

Table I: Integrated Polarized Distributions: $\Delta G=x G$ (above line), $\Delta G=0$ (below line)

$\begin{array}{cccccc}\text { Quantity } & S M C\left(I^{P}\right) & S M C\left(I^{d}\right) & E 154\left(I^{n}\right) & E 143\left(I^{d}\right) & \begin{array}{c}H E R M E S \\ \left(I^{n}\right)\end{array} \\ \langle\Delta u\rangle_{\text {tot }} & 0.75 & 0.73 & 0.75 & 0.72 & 0.74 \\ \langle\Delta d\rangle_{\text {tot }} & -.35 & -.37 & -.30 & -.31 & -.29 \\ \langle\Delta s\rangle_{\text {tot }} & -.05 & -.08 & -.02 & -.03 & -.01 \\ I^{p} & 0.136 & 0.129 & 0.134 & 0.131 & 0.135 \\ \langle\Delta q\rangle_{\text {tot }} & 0.35 & 0.28 & 0.43 & 0.38 & 0.44 \\ ------ & ---- & ----- & ------ & ----- & ---- \\ \langle\Delta u\rangle_{\text {tot }} & 0.73 & 0.70 & 0.73 & 0.70 & 0.72 \\ \langle\Delta d\rangle_{\text {tot }} & -.37 & -.39 & -.33 & -.33 & -.31 \\ \langle\Delta s\rangle_{\text {tot }} & -.07 & -.10 & -.04 & -.06 & -.04 \\ \langle\Delta q\rangle_{\text {tot }} & 0.29 & 0.21 & 0.36 & 0.31 & 0.37\end{array}$

From the results in Table I, we can draw the following conclusions:

(1) The naive quark model is not sufficient to explain the proton's spin characteristics, since the total quark contribution to proton spin falls between about $\frac{1}{4}$ and $\frac{1}{2}$. The experimental uncertainties and the uncertainty due to the choice of $\Delta G$ are comparable. 
(2) The total up and down quark contributions to proton spin all agree to within a few percent. The proton and deuteron data imply a larger polarized sea. The strange sea contribution is the most uncertain of all the flavors, but is definitely suppressed compared to the lighter quark flavors.

(3) This analysis is consistent with a small anomaly correction. Specifically, a larger anomaly term from a greater $\Delta G$ implies that the strange sea would be positively polarized, while the other flavors are negatively polarized. Since there is no obvious mechanism that allows selective polarization of different flavors, we conclude that these data imply that either: (i) $\Delta G$ is small or (ii) the factorization which separates out the anomaly from the total quark spin contribution and is gauge dependent, gives misleading results.

(4) The extracted value for $I^{p}$ is comparable for all the data and well within experimental uncertainties. This indicates the validity of the BSR.

These DIS experiments and the theoretical progress in calculating higher order QCD corrections have allowed us to narrow the range of the spin contributions for each flavor. They probe to smaller $x$ values, while decreasing the statistical and systematic errors. The main differences are the strange sea spin content and the size of $\Delta G$. There are a number of technologically feasible experiments that would supply more detailed information about these distributions. In-depth summaries can be found in reference [10].

\section{REFERENCES}

1. B. Adeva, et.al., Phys. Lett. B320, 400 (1994) and Phys. Lett. B369, 93 (1996); D. Adams, et.al., Phys. Lett. B329, 399 (1994) and Phys. Lett. B396, 338 (1997).

2. K. Abe, et.al., Phys. Rev. Lett. 74, 346 (1995), Phys. Rev. Lett. 75, 25 (1996) and hep-ex/9610007.

3. K. Ackerstaff, et.al., hep-ex/9703005.

4. M. Goshtasbpour and G. Ramsey, Phys. Rev. D55, 1244 (1997).

5. J.-W. Qiu, et. al., Phys. Rev. D41, 65 (1990).

6. S.A. Larin, Phys. Lett. B334, 192 (1994).

7. A.V. Efremov and O.V. Teryaev, JINR Report E2-88-287 (1988); R.D. Carlitz, J.C. Collins, and A.H. Mueller, Phys. Lett. B214, 229 (1988).

8. S.A. Larin, et. al., hep-ph/9702435.

9. E. Stein, et. al., Phys. Lett. B353, 107 (1995).

10. G.P. Ramsey, Particle World, 4, No. 3, (1995) and hep-ph/9702227. 